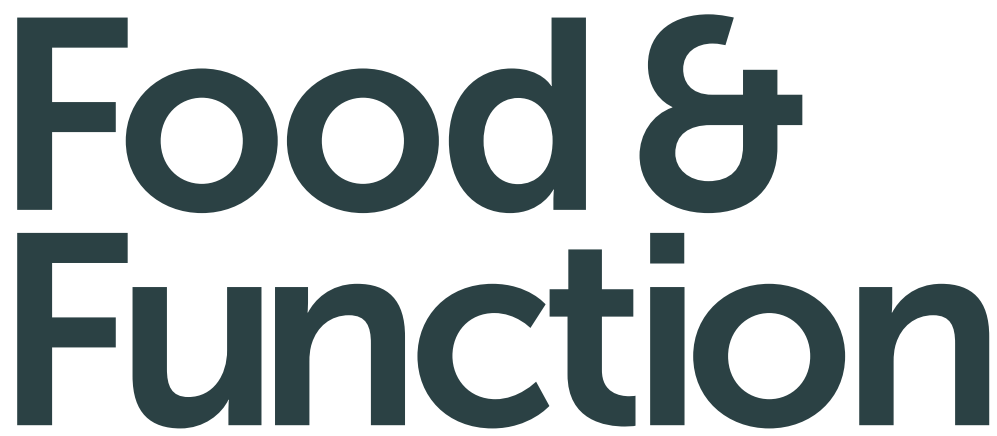

Linking the chemistry and physics of food with health and nutrition rsc.li/food-function
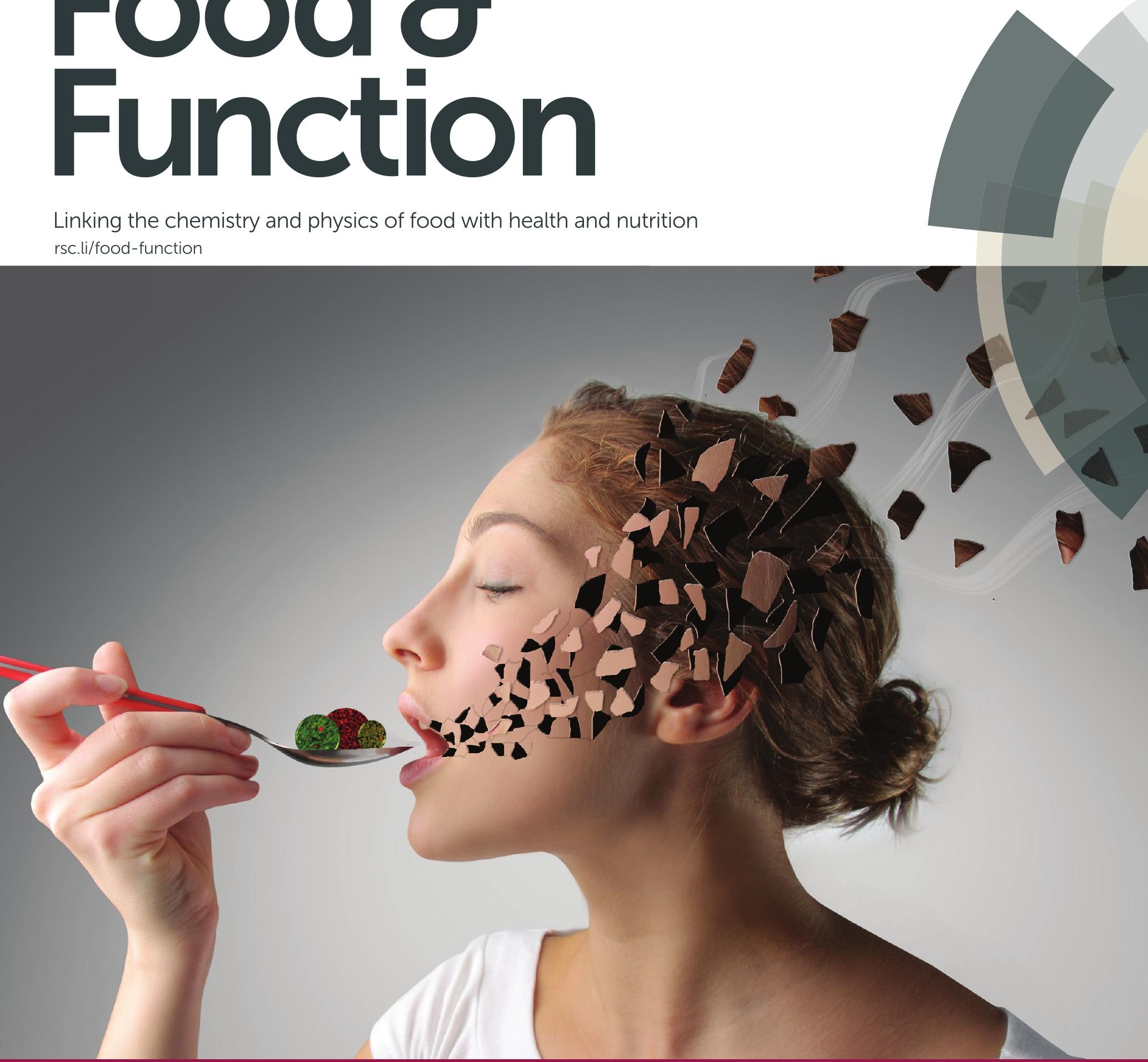

Themed issue: Structure \& Sensory

ISSN 2042-650X

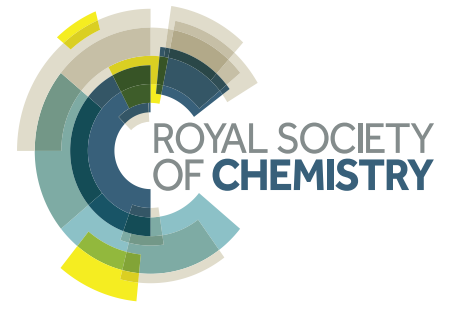




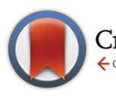

CrossMark

Cite this: Food Funct., 2017, 8, 464

\section{Bolus matters: the influence of food oral breakdown on dynamic texture perception}

\author{
Marine Devezeaux de Lavergne, ${ }^{a, b}$ Fred van de Velde ${ }^{a, c}$ and Markus Stieger ${ }^{a, b}$
}

This review article focuses on design of food structure, characterisation of oral processing by boli characterisation and dynamic texture perception. Knowledge of the food properties governing bolus formation and bolus properties determining temporal changes in texture perception is of major importance. Such knowledge allows academia to better understand the mechanisms underlying texture perception and food industry to improve product texture. For instance, such knowledge can be used for developing foods with desired texture perception that fit in a healthy diet or that are customized to specific consumer groups. The end point of oral processing is the formation of a safe-to-swallow bolus. The transitions of solid and soft solid foods into bolus are accompanied by tremendous modifications of food properties. The review discusses dynamic changes in bolus properties resulting in dynamic changes of texture perception during oral processing. Studies monitoring chewing behaviour are discussed to complement the relationships between bolus properties and dynamic texture perception. We conclude that texture perception evolves over mastication time and depends on food properties, such as mechanical properties, mainly in the beginning of oral processing. Towards the middle and end of oral processing, bolus properties depend on food properties and explain texture perception better than food properties.
Received 6th July 2016, Accepted 3rd September 2016 DOI: 10.1039/c6fo01005a rsc.li/food-function
The constant modifications of food structure during oral processing contribute to the complexity of texture perception. Such modifications are responsible for dynamic changes in texture perception, and are especially relevant for semi-solid and solid foods. Oral processing consists of processes occurring in the oral cavity and different stages can be discriminated, such as first bite, chew down and swallowing. The functions of oral processing are to recognise the food as being edible and to process it into a swallowable mass referred to as food bolus. ${ }^{9}$ The dynamic modifications of food properties during eating were introduced by Hutchings and Lillford by stating that "texture" depends on oral processing. ${ }^{10}$ As shown in Fig. 1, Hutchings and Lillford described different breakdown pathways for different food products. Together with oral processing time, the degree of structure and degree of lubrication were described as the main parameters contributing to bolus properties. A safe swallow requires to be below a maximum degree of structure and above a minimum degree of lubrication. The structure of foods is broken down by comminution by the teeth and the tongue, but also dissolution of components in saliva and, in the case of starch containing or starch based products, enzymatic starch breakdown by amylases. Increase of lubrication is reached by addition of saliva, release of fluids from the food matrix and phase transition of fat or other components caused by changes of temperature. ${ }^{8,11}$ Those modifications influence the physical properties of the bolus and thus its perception over oral processing time. ${ }^{12}$ 


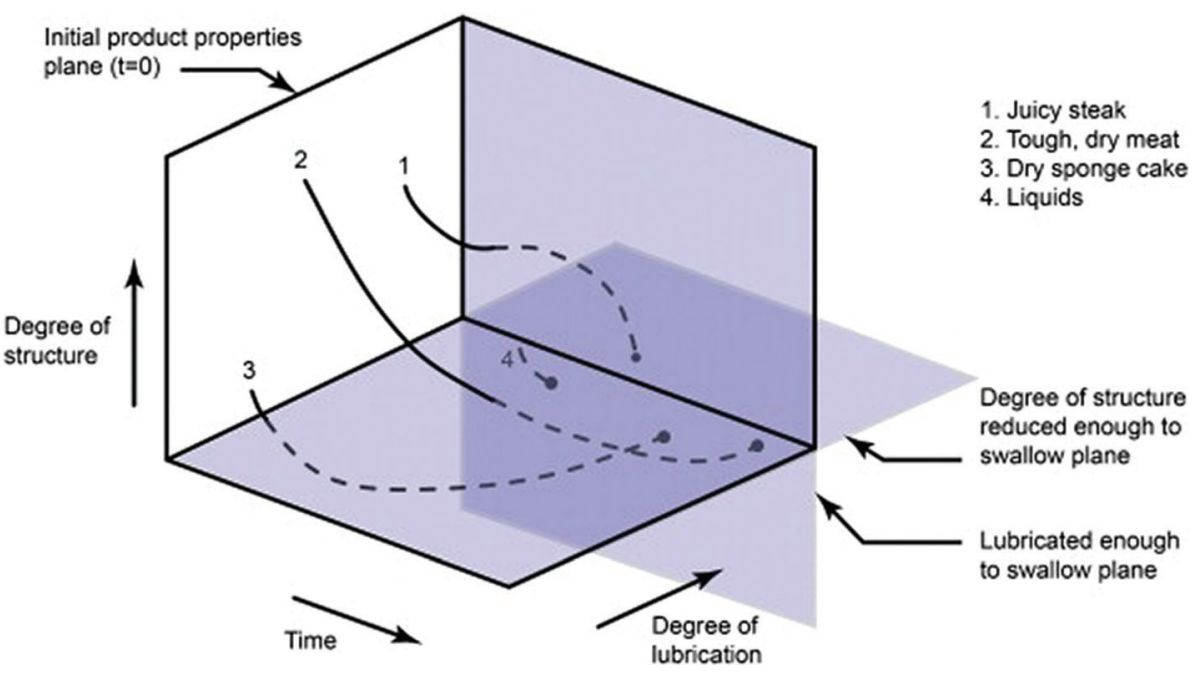

Fig. 1 Hutchings and Lillford's philosophy of the breakdown path. ${ }^{10}$

The bolus formation as well as the impact of food properties on bolus formation has been studied. Sampling methods, which consist of collecting and characterizing the broken down bolus ex situ, can be used to observe changes in bolus properties during mastication. ${ }^{13} \mathrm{~A}$ wide range of methodologies have been developed to characterize the bolus breakdown and lubrication properties. ${ }^{14-18}$ Panouillé et al. provided an extensive review of experimental methods applied for bolus characterization and discussed the advantages and limitations of the methods. ${ }^{19}$ The variety of characterization methods demonstrates the need to adapt the method to the specific properties of the bolus, which can differ considerably depending on the type of food and the degree of breakdown. All methods contributed to a better understanding of bolus formation, even though ex situ measurements have been suggested to be replaced by in situ measurements to improve time accuracy. ${ }^{19}$ Overall, the link between bolus formation and dynamic texture perception remains unclear and requires a systematic approach.

On the one hand, food scientists tend to investigate structural and rheological properties of foods in relation to texture perception and often disregard measurements of oral processing. On the other hand, dentists and biologists tend to investigate the physiology of eating by measuring oral physiological parameters. ${ }^{20}$ The integration of the different scientific disciplines is seldom found in literature. Due to the complexity of texture perception, a multidisciplinary approach is required to investigate the relationships between food and bolus properties and texture perception. The three main research areas are (a) evaluation of texture by sensory research, (b) studies of the relationships between food structure and mechanical properties and (c) studies of food breakdown in relation to oral physiology, which can be split further into studies investigating bolus properties and studies focusing on oral physiology. ${ }^{12}$ Recent studies focused on understanding the links between oral processing and dynamic texture perception of breads, cheeses, biscuits and gels and are discussed in this review. First, the measurement of dynamic texture perception, from ingestion to swallow, by different sensory methods is discussed in this review. Secondly, the impact of food properties on texture perception, which allows to control texture perception by food structure design, is reviewed. Thirdly, the involvement of food oral processing in dynamic texture perception is discussed to fill the gaps between structure design and texture perception. Fourthly, an outlook on industrial implications and future research is given.

\section{Role of food structure in dynamic texture perception}

\subsection{Assessment of dynamic texture perception}

Assessing texture by sensory methods can be done using a variety of sensory methodologies. The most commonly used methods are texture profiling methods and time resolved sensory methods.

2.1.1. Texture profiling. The classification of textural characteristics began with the developments of texture profile analysis $^{21,22}$ where terms describing sensory texture were classified into three groups according to their common physical meaning: mechanical, geometrical and other characteristics, such as fat and moisture. Texture profile analysis assesses the degree in which a texture term is perceived using an intensity scale and the order of appearance principle. In Table 1, the classification of texture terms from Brandt et al. in the order of appearance is summarized. ${ }^{22}$ Table 1 shows the texture terms commonly used in literature in relation to food properties. It can be seen that texture perception changes dramatically in time, with attributes being perceived at first bite, followed by early chew-down, late chew-down, swallow and after swallowing (residual). ${ }^{20,23}$ Specific texture attributes, such as firm or crunchy, are typically perceived at first bite, whereas 
Table 1 Texture profiling scheme adapted from Brandt et al., ${ }^{22}$ supplemented by the classification of textural characteristics from Szczesniak ${ }^{21}$ (white columns), and additional sensory terms reviewed by Pascua et al. ${ }^{23}$ (grey columns)

First bite

\begin{tabular}{|c|c|c|c|}
\hline \multicolumn{2}{|l|}{ Mechanical } & \multicolumn{2}{|l|}{ Geometrical product dependant } \\
\hline Fracturability & Crumbly, crunchy, brittle & & \\
\hline \multicolumn{4}{|c|}{ Chew down (early) } \\
\hline \multicolumn{2}{|l|}{ Mechanical } & \multicolumn{2}{|l|}{ Geometrical product dependant } \\
\hline Fracturability & Crumbly, crunchy, brittle & Particle size and shape & Gritty, grainy, coarse \\
\hline Gumminess & Short, mealy, pasty, gummy & & \\
\hline Chewiness & Tender, chewy, tough & & \\
\hline Adhesiveness & Sticky, tacky, gooey & & \\
\hline Springiness & Plastic, elastic & & \\
\hline
\end{tabular}

Chew down (late)

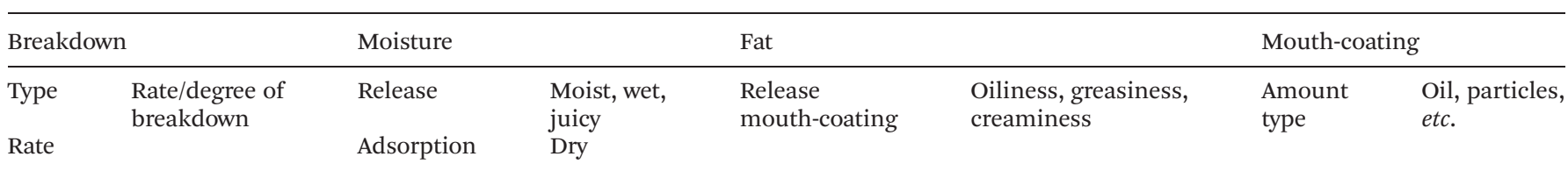

Residual (after swallow)

\begin{tabular}{llll}
\hline Mouth-coating & & Throat-coating & General feel on \\
\hline Type & Oil, moisture, particles, etc. & Type & Mouth \\
Amount & & Amount & Throat
\end{tabular}

different attributes, such as juicy or creamy, are typically perceived during late chew down.

Texture profile analysis is the only intensity scaling sensory method focusing solely on texture. However, other conventional sensory techniques such as Quantitative Descriptive Analysis (QDA) can be used to assess food texture by using texture profile analysis terms or to assess food texture, flavour and appearance simultaneously. ${ }^{12,24}$ Even though dynamic aspects of texture are acknowledged in these sensory methods by the order of appearance principle, they require an integration of perception of each attribute over time to a single intensity value. Therefore, these descriptive, static sensory measures describe dynamic changes in texture perception to a limited extent. $^{25-27}$

2.1.2. Time resolved sensory methods. In contrast to descriptive sensory methods, such as the texture profile method and QDA that pre-determine the time of rating the intensity of a texture attribute by providing instructions to the subjects, time resolved sensory methods allow to continuously evaluating sensory attributes during eating. Time intensity (TI) methods continuously track the intensity of one, or in a few studies two, attributes over time. ${ }^{25}$ Using discrete tracking, progressive profiling has also been used to describe the intensity of a few attributes at specific time points over oral processing. ${ }^{28}$ Progressive profiling has been used to correlate texture perception to bolus properties over oral processing time. ${ }^{29}$
More recently, Temporal Dominance of Sensations (TDS) was developed as a time resolved method focusing on the determination of the most "dominant" sensation over time. ${ }^{30}$ The dominant sensation is often defined as the sensation that catches the most attention at a time point during consumption. TDS can rate up to 10 sensory attributes in one test, which can be texture related or a combination of aroma, taste and texture attributes. TDS requires little training of panellists, which is a considerable advantage over TI. However, compared to QDA, the choice of attributes is limited and crucial. Indeed, the selection of attributes used in TDS influences the outcomes. ${ }^{31}$ TDS is an effective method to demonstrate the sequence of sensations over time. ${ }^{30}$ Still few studies using TDS and focusing on texture perception have been conducted. Lenfant $e t$ al. used TDS to study breakfast cereals with a focus on dynamic texture perception. ${ }^{32}$ This study demonstrated the importance of dynamic sensory methods in texture perception that can be expressed as sensory trajectories. It was shown that different attributes were perceived as dominant at the beginning, such as hardness and crunchiness, the middle, such as brittleness and lightness, and the end phase of mastication, such as stickiness.

Sequences of dominant texture attributes were found in other studies focusing on biscuits, breads, cheeses and gels. In biscuits varying in sugar and fat content, hard was perceived as dominant sensation in the beginning of mastication 
followed by crunch/crispy and crumbly in the middle of mastication and dry followed by sticky towards the end of mastication. ${ }^{33}$ Panouille et al. studied breads varying in density and fat content using TDS. ${ }^{34}$ Breads were perceived with a congruent succession of dominant attributes with hard or soft being dominant first, followed by aerated or dense and finally hydrated. Some attributes, such as crumbly, dry or sticky, were perceived at various time points as dominant depending on the composition of the bread. It can be seen in Fig. 2 that the sensory trajectories of breads varying in density and fat content converge at the end of mastication. Commercial cheeses varying in moisture and fat content also showed a consistent sequence of dominant attributes. ${ }^{35}$ Soft was perceived initially for all cheeses as dominant followed by "brittle gel" in low fat cheeses and sticky in all cheeses. Residual film and mouth coating were dominant towards the end of mastication. The attribute fat appeared in the middle or the end of mastication depending on the fat content of the cheeses. For emulsion-filled gels, firm was perceived at the beginning of oral processing as dominant followed by elastic and sticky or moist and refreshing depending on the fracture strain of gels. At the end of oral processing the gels were perceived melting and grainy or creamy depending on fracture strain of the gels. ${ }^{29}$ In another study using gels with mechanical contrast, a similar succession of attributes occurred but grainy and melting were dominant during the middle and end of oral processing. ${ }^{36}$

Recently, Temporal Check-All-That-Apply (TCATA) has been introduced as a method to quantify dynamic sensory percep-
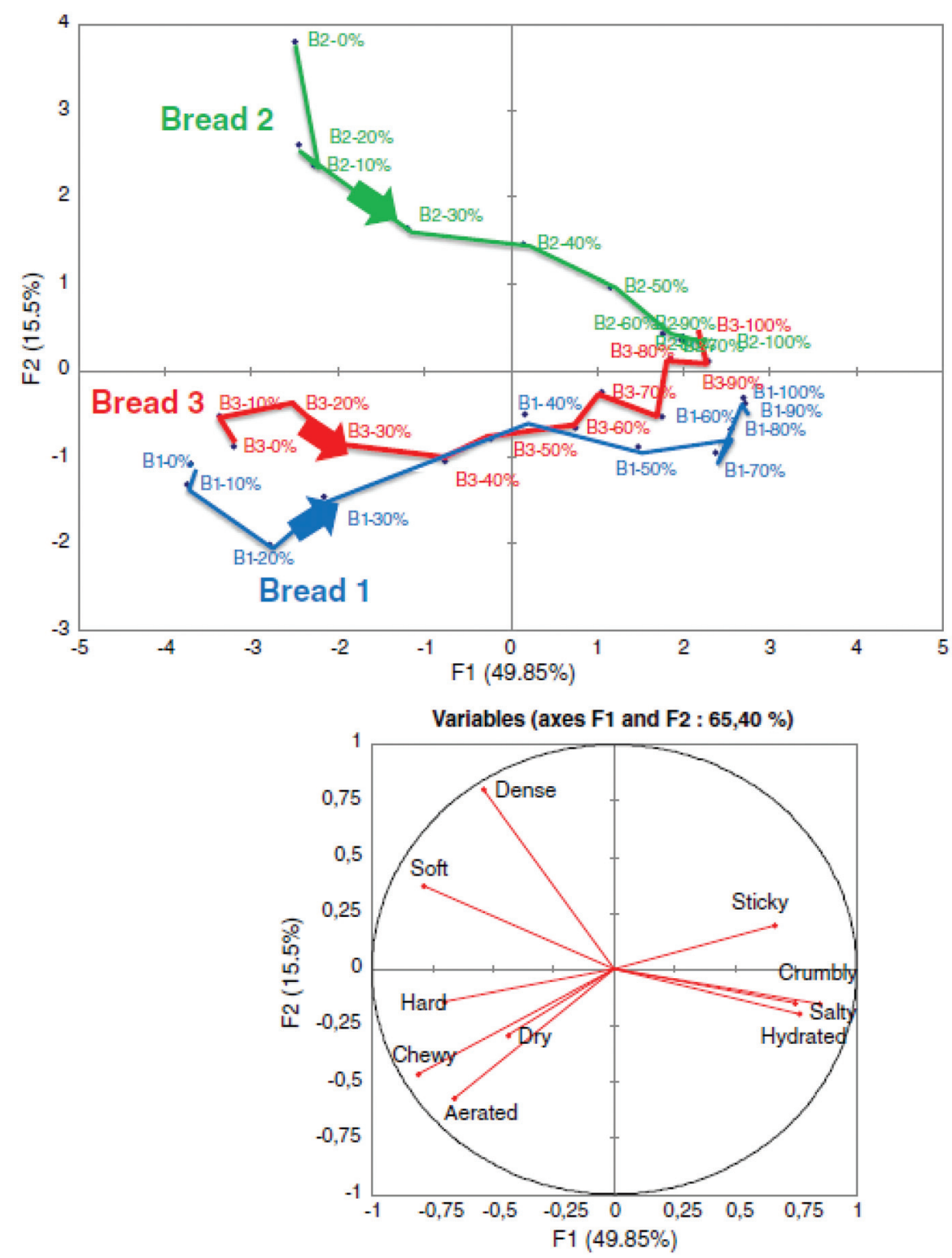

Fig. 2 PCA on dominance values derived from TDS data for three breads differing in density: Bread 1 is the reference bread, Bread 2 is the dense bread and Bread 3 is the bread with added fat. ${ }^{34}$ 
tion of foods. ${ }^{37-39}$ In TCATA, subjects select the attributes they consider applicable to describe the sensations they perceive from a list of attributes. The assessment is performed at different moments in time during oral processing. TCATA has been demonstrated to quantify the dynamic sensory perception of a variety of products. It has been suggested that TCATA may provide a more detailed description of dynamic sensory perception compared to TDS. ${ }^{40}$ To the best of our knowledge, TCATA has not been used yet in studies linking dynamic sensory and texture perception to food oral breakdown, bolus properties and oral processing behaviour.

The variety of sequences of dominant sensations obtained using TDS shows the influence of the products on dynamic texture perception. However, several attributes appear at consistent time points regardless of the type of product, such as firm/soft in the beginning of oral processing and fat related attributes towards the end. This order of appearance is in agreement with the pre-defined order of appearance used in texture profile analysis.

2.1.3. Comparing descriptive and time resolved sensory methods. By comparing QDA with TDS, a few studies demonstrated that the results were highly correlated. While QDA offers to rate many texture attributes, TDS brings a time dimension to the sensory evaluation. ${ }^{29,41,42}$ QDA requires an extensive training of the panellists and longer evaluation sessions than TDS but provides a more detailed sensory profile including information on taste and aroma. Often TI methods are used to complement descriptive methods, as they provide different types of information. ${ }^{41,43}$ In the case of oral processing studies, the interest of using TDS was emphasized by Cheong et al. ${ }^{44}$ Even though performing TDS modifies eating behaviour slightly, the natural differences in chewing behaviour observed between products were preserved while performing TDS. ${ }^{44}$ Moreover, TDS is a relatively new sensory method that is still being developed. For instance, TDS was recently used to assess sensory properties of foods over multiple bites/ sips. ${ }^{45}$ Multiple bite/sip studies using TDS allow to follow changes in perception over a longer consumption time i.e. when an entire meal is consumed. This could be of interest in future studies related to oral processing behaviour to monitor changes in dynamic texture perception not only per bite but also from the beginning of consuming a meal to the end.

\subsection{Role of mechanical properties in texture perception}

Mechanical properties are a major factor contributing to food texture and can be measured under small or large deformation. Large deformation rheology is closer to the conditions occurring during mastication as the food deforms considerably and fractures. To describe the response of food materials to deformation, the two properties used are stress $(\sigma)$ and strain $(\varepsilon)$. Stress represents the force applied to a unit area and strain represents the deformation per unit length. As one of the main goals of mastication is to fracture foods to obtain a bolus that is safe to swallow, fracture stress and strain are two descriptors of food mechanical properties that are frequently found in oral processing studies. In addition, other mechan- ical properties such as Young's modulus or recoverable energy are widely used. ${ }^{46,47}$ The impact of food properties on dynamic texture perception is summarized in Table 2 and discussed in the following.

2.2.1. First bite texture perception. The most evident correlation between food mechanical properties and texture perception is the link between instrumentally measured mechanical hardness and perceived hardness/firmness at first bite. Mechanical hardness can be measured in different ways depending on the food product analysed. The derived parameters, such as fracture stress, critical stress intensity or Young's modulus, are linked to the force used to compress a material between the teeth. ${ }^{20}$ This relationship was evidenced in fruits and vegetables, ${ }^{3,4}$ cheeses, ${ }^{48-50}$ biscuits ${ }^{51}$ and gels. ${ }^{2,8,52,53}$ Fracture strain was linked to perceived deformability and brittleness at first bite ${ }^{2,29,52}$ and fracturability ${ }^{53}$ in gels and cheeses. However, correlations between fracture properties and first bite perception are not always observed. van den Berg et al. suggested that other rheological properties such as the shape of the post-fracture curve or surface properties also contribute to texture perception of first bite attributes of mixed whey protein-polysaccharide gels. ${ }^{47}$

2.2.2. Chew down texture perception. The relationship between fracture properties of model foods and texture perception during chew down was discussed in literature but remains unclear. ${ }^{53-55}$ After first bite, mechanical properties obtained from large deformation measurements are often difficult to correlate with texture perception. Moreover, they are time-rate dependent and vary with test conditions. ${ }^{56}$ Moreover, mechanical properties of gels are convoluted. Young's modulus and fracture energy have been correlated to hardness or fracturability during chew down. ${ }^{56}$ Recoverable energy of gels correlated to perception of crumbly ${ }^{46,47}$ and perceived cohesiveness, adhesiveness and moisture release. ${ }^{55}$

Gwartney et al. used emulsion filled gels from stranded and particulate whey protein networks to design gels varying in fracture properties. ${ }^{53}$ Fracture stress was positively correlated to perceived firmness, whereas fracture strain correlated to several chew down texture attributes. For instance, gels with a high fracture strain were perceived smoother, with larger and less cohesive particles and less crumbly than gels with low fracture strains. However, fracture strain was correlated to water holding capacity, which also correlated to several chew down texture attributes, including moisture perception. Using emulsion-filled gels, Sala found that fracture properties impact creaminess. ${ }^{57}$ In gels with different structural as well as fracture properties, Çakir et al. found similar results, such as a high cohesiveness perception and high residual particle mouth coating in low fracture strain gels. ${ }^{55}$ Devezeaux de Lavergne et al. discussed the link between fracture properties and chew down texture perception in emulsion-filled gels. ${ }^{29,58}$ As shown in Fig. 3, gels with a high fracture stress were perceived as firm and not spreadable during chew down. Gels with a low fracture strain were perceived powdery and grainy during chew down and with a high amount of residue after expectoration. Usually, fracture stress and strain both influ- 
Table 2 Sensory attributes and related oral processing and rheology/fracture properties, completed from Koç et al. ${ }^{56}$ This table does not indicate correlations between rheology, chewing behaviour and bolus properties. TCPT (two cycle penetration test) was developed by Devezeaux de Lavergne et al. ${ }^{36}$ based on an inverse TPA with redefined parameters that describe physical properties of the boli. (-) indicates negative correlations between sensory terms and other parameters

Early chew down texture attributes (first chews)

\begin{tabular}{llll}
\hline Sensory attribute & Rheology/fracture & Chewing behaviour & Bolus properties \\
\hline Hardness & Fracture stress & Jaw-closing muscle activity & TCPT first peak force \\
Firmness & Maximum compliance creep & Jaw movements & TCPT flowability (-) \\
& Young's and fracture modulus & Chewing velocity & Number of chews \\
& Work of fracture & Chewing time & \\
Stress intensity factor & Saliva incorporation & Number of chews \\
Fracturability & Fracture surface energy & Chewing time & \\
Brittleness & Fracture strain & & \\
Crumbliness & Recoverable energy & Occlusal duration & \\
Deformability & Held water & & \\
\hline
\end{tabular}

Middle chew down texture attributes

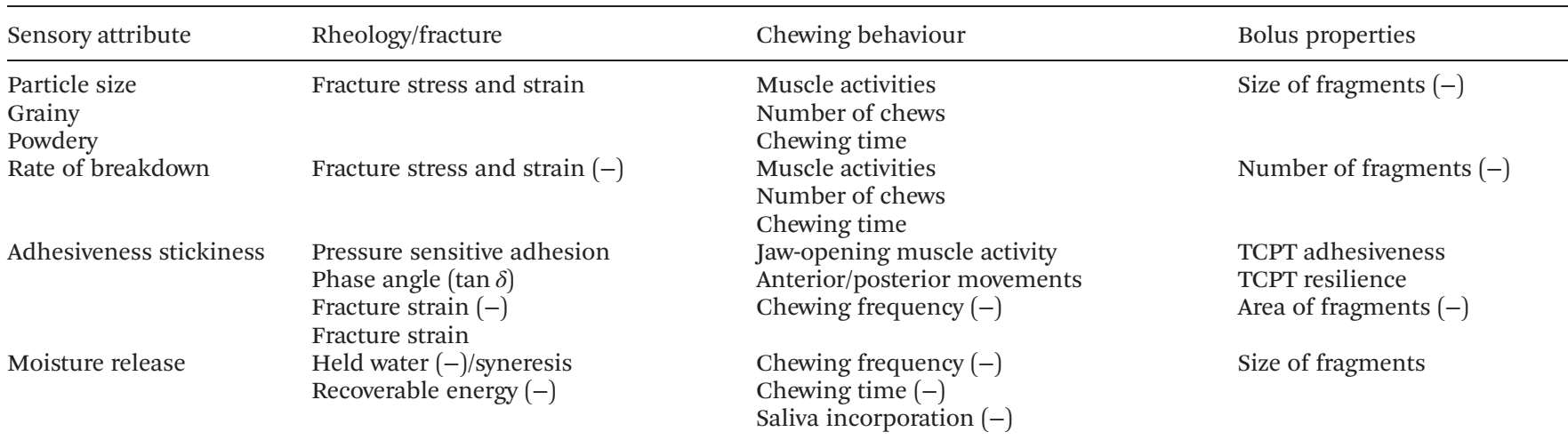

Late chew down texture attributes

\begin{tabular}{|c|c|c|c|}
\hline \multirow[t]{2}{*}{ Cohesiveness } & Recoverable energy (-) & Number of chews $(-)$ & \\
\hline & Fracture strain $(-)$ & Chewing time $(-)$ & \\
\hline \multirow[t]{3}{*}{ Creaminess } & Fracture strain & & TCPT flowability \\
\hline & Fracture stress $(-)$ & & Number of fragments $(-)$ \\
\hline & Melt below $37^{\circ} \mathrm{C}$ & & \\
\hline
\end{tabular}

Residual texture attributes

\begin{tabular}{llll}
\hline Sensory attribute & Rheology/fracture & Chewing behaviour & Bolus properties \\
\hline Particle mouth coating & Recoverable energy $(-)$ & $\begin{array}{l}\text { Jaw and tongue movements } \\
\text { Frequency }(-)\end{array}$ & Number of fragments \\
& Fracture strain $(-)$ & $\begin{array}{l}\text { Chewing time } \\
\text { Jaw and tongue movements } \\
\text { frequency }(-)\end{array}$ & \\
Moisture mouth coating & Held water $(-)$, syneresis & Saliva incorporation $(-)$ &
\end{tabular}

enced perception of chew down texture attributes. For instance, gels combining a low fracture stress and a high fracture strain were perceived fatty, melting and creamy during chew down. ${ }^{29,58}$ Some differences in texture perception could be seen as the gels differed between the studies. In general, results are congruent in literature and confirm the impact of fracture properties on chew down texture perception.
TDS sensory trajectories showed that fracture stress mainly impacted early chew down whereas fracture strain impacted late chew down with gels having a high fracture strain being perceived creamier and less grainy. ${ }^{29}$ In Devezeaux de Lavergne et al., fracture stress of agar and gelatine emulsion-filled gels was varied. ${ }^{59}$ Gels with low fracture stress were perceived dominantly melting and refreshing in the middle of oral processing 


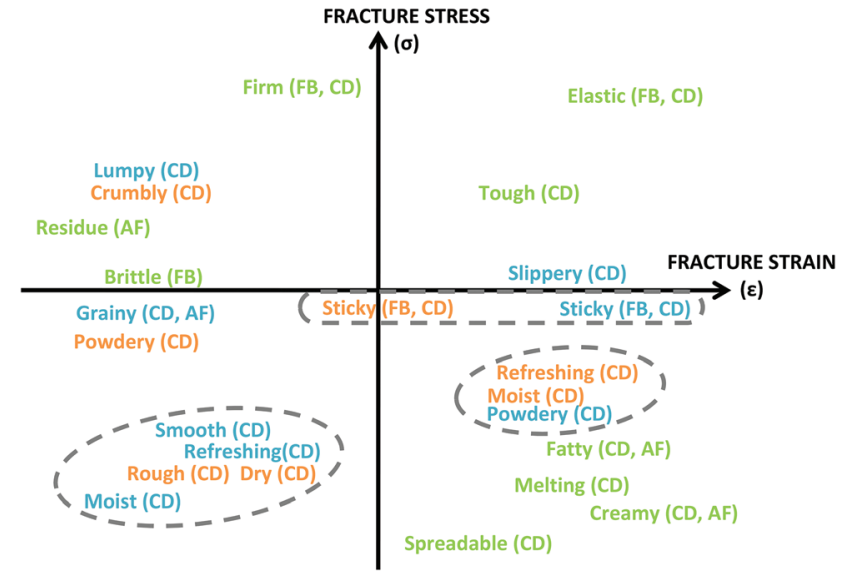

Fig. 3 Relation between fracture properties and texture attributes perceived at first bites (FB), chew down (CD) and after-feel (AF) of model gels. This figure was built as simplified attribute loadings on a PCA, based on a visual compilation of PCA plots obtained from two studies where fracture properties determined both PC1 and PC2: texture attributes common from Devezeaux de Lavergne et al. ${ }^{29,58}$ are in green, attributes from Devezeaux de Lavergne et al. ${ }^{29}$ are in blue and from Devezeaux de Lavergne et al. ${ }^{58}$ are in orange. Circled with dotted lines are attributes used in Devezeaux de Lavergne et al. ${ }^{29,58}$ that differed between studies.

and creamy at the end of oral processing. Gels with a high fracture stress were perceived dominantly firm in the beginning of oral processing and grainy in the middle of oral processing. These results confirm that fracture stress is mainly impacting perception at the beginning and middle, and has only a slight impact on texture perception at the end of oral processing. Fracture strain is expected to change breakdown of gels to a greater extent than fracture stress. Indeed, small movements in the mouth are sufficient to break gels with low fracture strain whereas changes in fracture stress can be compensated for by chewing with a higher muscle force. ${ }^{60}$ An interesting finding is the impact of fracture strain on creaminess perception, as creaminess is a fat related attribute highly relevant for the development of fat reduced foods.

2.2.3. Residual texture perception. The perception of residual texture attributes is the last stage of texture perception (Table 1) and results from the presence of particles or residues adhering to the oral tissues after swallowing the main bulk of the bolus, also referred to as oral coatings. Mechanical properties of food have been shown to impact the perception of residual texture attributes, such as a high residual particle mouth coating in low fracture strain gels. ${ }^{55}$ The dominant residual texture attributes of gels were found to be congruent with perception at the end of oral processing (before swallow) and mainly depending on fracture strain (Devezeaux de Lavergne et al. 2015a). ${ }^{29}$ A study by Camacho et al. focused on the residual perception of emulsion-filled gelatine gels. ${ }^{61}$ Camacho et al. found that a higher fat content in gelatine gels as well as droplets being unbound to the gel matrix both result in a more fat being deposited on the tongue as well as a higher perception of fatty after-feel. ${ }^{61}$ Moreover, they showed that fatty after-feel decreased as fat oral coatings were cleared from the tongue.

\subsection{Additional food properties impacting texture perception}

Rheological properties of food depend on food composition and microstructure, which are known to impact other food properties such as surface properties. Moreover, variations in these properties might have a distinct impact on texture perception. Often, rheology, composition and structure are convoluted. Thus, the role of food composition, structure and resulting food properties including rheological, surface and tribological properties, should be considered in texture research.

Besides the differences in mechanical properties, it was shown that specific properties of ingredients impacted chew down texture perception. In Devezeaux de Lavergne et al., ${ }^{58}$ composition accounted for $48 \%$ and fracture properties for $47 \%$ of the variation in texture perception of emulsion-filled gels made of agar/gelatine, carrageenan/locus bean gum and high/low acyl gellan. In a study by Sala et al., ${ }^{54}$ it was found that texture attributes, such as crumbly, slippery and melting differed between emulsion-filled gels made of whey protein, gelatine and different carrageenans. These results suggest that the composition of gels is as relevant as their mechanical properties for texture perception. However, the differences in perception due to composition are mainly perceived during chew down. Fracture properties still dominate perception at first bites.

The impact of food composition on texture perception during chew down can originate from differences in microstructure and differences in physical-chemical properties. Microstructure, when uncoupled from fracture properties, was found to have an impact on texture perception in mixed protein-polysaccharide gels. ${ }^{5,7,62}$ Differences in microstructure, such as pore size and micro-phase separation, impacted serum release from gels. ${ }^{63}$ Serum release strongly influences moisture related sensory attributes, such as juiciness and wateriness, which are perceived during first chew ${ }^{4,53,55}$ and during chew-down. ${ }^{62}$ In Devezeaux de Lavergne et al. ${ }^{29,58}$ the differences in serum release between gels, namely agar/gelatine gels not showing serum release and carrageenan/LBG and high/low acyl gellan gels showing serum release, could explain the differences in moist and refreshing perception (Fig. 3).

In emulsion-filled gels, a higher fat content and smaller fat droplets enhance creaminess perception. ${ }^{57}$ In addition, oil droplets being unbound to the gel matrix typically increase creaminess perception, as the oil can be released upon fracture and coalesce in the oral cavity. ${ }^{64,65}$ However, this effect is not always found. ${ }^{64}$ Moreover, oil binding to the gel matrix is known to affect fracture properties of gels. Unbound oil droplets act as inactive fillers and reduce the Young's modulus, whereas bound oil droplets, which act as active fillers, increase the Young's modulus with increasing oil concentration when the modulus of the droplets is larger than the modulus of the matrix. ${ }^{66}$ In literature, binding is often varied by changing the emulsifier and maintaining the properties of the gel matrix, 
which results in gels with unbound droplets having different fracture properties than gels with bound droplets. ${ }^{6}$ In Devezeaux de Lavergne et al., ${ }^{29}$ changes in gel matrix were compensated for by adjusting the composition to obtain gels with bound and unbound droplets with similar fracture properties. No significant differences in creaminess or in any other texture attribute between gels having bound and unbound droplets and similar fracture properties were observed. These results suggest that the effect of oil binding on texture perception might be negligible compared to the effect of fracture properties on texture perception. It is therefore difficult to conclude that oil binding has a significant impact on texture perception.

Besides microstructure, phase transitions of food ingredients can have a large impact on chew down texture perception. "Melting" is a sensory attribute related to food breakdown, which can be perceived during late chew down. Sensory melting can result from liquefaction of starch upon hydrolysis by amylases present in saliva ${ }^{67}$ or from physical melting of the gel matrix due to temperature changes occurring during oral processing. For example, gelatine starts to melt below mouth temperature, ${ }^{68}$ which increases creaminess in emulsion-filled gels. ${ }^{29,54}$ Sala et al. concluded that the melting behaviour of gels dominated sensory perception compared to fracture properties. ${ }^{54}$ In addition, agar/gelatine gels generated more friction than carrageenan/LBG and gellan gels, which correlated to a higher sticky sensation, especially for gels with high fracture strain containing more gelatine. ${ }^{29}$ Therefore, other properties of food such as the composition, serum release and tribological properties, next to fracture properties have to be taken into account when discussing texture perception.

\section{Role of oral processing in dynamic texture perception}

The role of oral processing as the missing link between dynamic texture perception and food and bolus properties is more and more acknowledged and has recently been reviewed. ${ }^{69}$ Two approaches can be taken: studying bolus properties to correlate them to dynamic texture perception or studying foodbody interactions to better understand the physiology of texture perception. Both research approaches are connected.

\subsection{Bolus properties and its link to dynamic texture perception}

The impact of the bolus properties on dynamic texture perception is summarized in Table 2 and discussed below.

3.1.1. Bolus fragmentation. Fragmentation by comminution is the main factor decreasing structure of soft solid and solid foods during oral processing. The decrease in degree of structure by fragmentation can be quantified by the particle size distribution of bolus fragments obtained by sieving or image analysis. ${ }^{14,15,70-72}$ The particle size and spread of particles decreased while the number of particles increased with oral processing time for bread boli. ${ }^{34}$ Devezeaux de Lavergne et al. observed in gels and sausages that with increasing eating time, number and total area of boli fragments increased and size of fragments decreased..$^{36,58,59,73}$ Roundness of fragments increased with oral processing time..$^{59,73}$ Similarly, in gel foods varying in textural complexity, the size of particles decreased and number of particles increased in the boli with mastication time. Gels displaying high textural complexity contained a several structural elements differing considerable in rheological and physical-chemical properties. The number of particles increased faster and more steadily in gels with high textural complexity, than in gels showing a lower textural complexity. ${ }^{74}$ These results demonstrate that the formation of a safe-toswallow bolus requires breakdown of the food in small and numerous fragments. Oppositely, in bolus from biscuits, agglomeration was observed with increasing mastication time rather than separation of the broken down particles. Agglomeration coincided with the perception of a paste-like biscuit rather than the perception of individual particles. ${ }^{75}$ This suggests that dry foods need to be broken down and agglomerated by saliva to be swallowed, whereas foods with a higher water or fat content mainly need to be broken down in smaller pieces to be safely swallowed. We conclude that breakdown patterns and pathways strongly depend on the type of food and its properties.

Breakdown patterns directly depend on food properties. Lillford observed that fracture properties were the most relevant rheological property of food during oral breakdown. ${ }^{76}$ Gels with high fracture strain were found to break down in few, large pieces at a slow rate. ${ }^{7,36,53,58,59}$ Gels with a low fracture strain break down into more, smaller fragments which have been shown to influence the dynamics of taste perception. ${ }^{77}$ Fracture stress was shown to have an impact on bolus formation. When comparing boli at swallow of foods widely varying in hardness, harder foods resulted in boli with smaller particles than softer foods. ${ }^{15}$ However, fracture stress of gels did not influence particle size and number in boli masticated for fixed time periods. ${ }^{36,58}$ These results suggest that subjects adapt their chewing force and frequency ${ }^{60}$ to break down hard and soft foods. The small particle size of boli of hard foods observed by Chen et al. ${ }^{15}$ could be due to longer chewing times resulting in smaller particles at the moment of swallow. Other food properties such as fat content were found to impact particle size distribution of boli. Low fat content of cheeses was associated with a high particle size in the bolus, even though low fat cheeses had a similar hardness as high fat cheeses. ${ }^{35}$ However, such results could be intertwined with changes in other mechanical properties of low fat cheeses or eating duration of the cheeses.

Particle size distribution of boli can be influenced not only by fragmentation but by more phenomena such as melting or dissolution. For instance, gels containing a mixture of agar and gelatine break down differently from gels composed of gellan gum or carrageenan/LBG due to melting of gelatine at mouth temperature. ${ }^{58}$ Boli of agar/gelatine gels contain smaller and rounder fragments than boli of other gels, probably as the agar/gelatine fragments melt in the mouth. Similarly, pure gelatine gels were shown to first break down in 
an increasing number of particles followed by a decrease in number of particles. ${ }^{59}$

Gel boli containing many small particles were perceived grainy or powdery. ${ }^{36,58}$ As gels with low fracture strain are broken down in many, small particles, they are perceived as grainy towards the end of oral processing. This coincides with TDS results on gels varying in fracture stress and strain. Gels separated in two groups at the end of the sensory trajectories, one group of high fracture strain gels that are perceived creamy and one group of low fracture strain gels that are perceived grainy. ${ }^{29}$ To conclude, the main factors influencing fragmentation of foods are fracture properties and melting behaviour.

3.1.2. Bolus lubrication. When considering the lubrication properties of the bolus, several aspects including saliva and dry matter content ${ }^{17}$ and the release of oil from the food matrix during mastication ${ }^{70}$ should be taken into account. The most evident factor that increases boli lubrication during oral processing is saliva incorporation. Saliva incorporation into the bolus is often quantified by gravimetrical analysis. ${ }^{78}$ Commonly, saliva content increases with increasing oral pro- cessing time. ${ }^{34,36,79}$ A low dry matter content of cheeses and gels was associated with more saliva incorporation into the bolus. $^{35,36,79}$ These results suggest that a target value of moisture content is required to safely swallow boli of soft solid foods. Saliva not only increases moisture content in boli, but also lubricates the boli by lowering friction. Saliva shows lower friction forces (better lubrication) than water due to the presence of salivary proteins. ${ }^{80}$ Reduction of friction by saliva is often overlooked in tribological studies. ${ }^{81}$

Similarly to the reduction of bolus friction by saliva addition, bolus lubrication can be increased by the release of fluids, such as oils, from the food matrix during oral processing. This was observed in model dairy products where a higher fat content resulted in less saliva incorporation into the bolus. $^{82}$ Similarly, gels with low serum release showed a higher friction force and incorporated more saliva. ${ }^{58}$ In emulsion-filled gels, oil release into the bolus during mastication was observed only in gels with unbound oil droplets to the gel matrix and was influenced by fracture properties, ${ }^{36,64}$ as illustrated in Fig. 4. Release of oil in unbound gels correlates to the

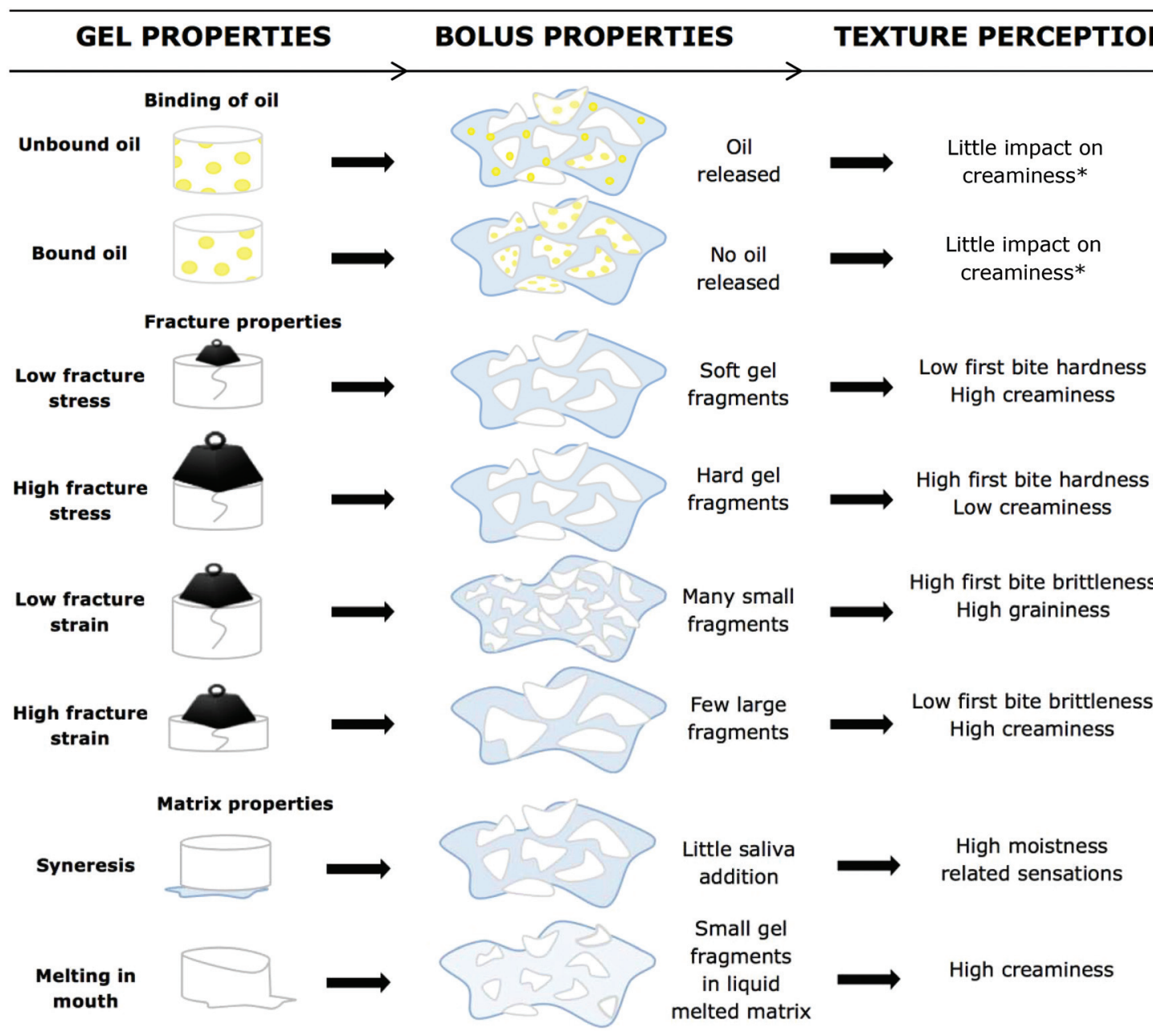

Fig. 4 Impact of gel properties on breakdown and subsequent texture perception. ${ }^{*}$ The effect of oil release on creaminess perception remains unclear in the current literature. 
total surface area of the gel fragments in the boli, suggesting that more oil is released as more surface is generated by the breakdown of gel particles. However, release of oil does not seem to impact perception of fat related attributes. ${ }^{36} \mathrm{~A}$ possibility is that most of the released droplets remain in the bulk of the bolus, thus they do not contribute to perception of fat related attributes. Moreover, fracture properties could overrule the relatively small effect of oil droplets release on perception of fat related attributes.

Incorporation of saliva into the food bolus also plays a role in degrading the structure of foods by dissolution and enzymatic breakdown of starches. Le Bleis et al. observed that bread boli masticated in a sealed bag had very similar mechanical properties to those of bread suggesting that water uptake from saliva plays a major role in the destructuration of bread. ${ }^{83}$ Moreover, the amylases present in saliva liquefy starch, which increases bolus lubrication. Janssen et al. found that melting sensations were related to thinning of starch based custards by enzymatic breakdown of starch and accompanied by a reduction in friction. ${ }^{67}$

Liquefaction of the food structure, similarly to enzymatic breakdown, also occurs by phase transitions of the food in the mouth due to changes of temperature. A recognizable example is melting of ice cream in the mouth. Similarly, gelatine gels first break down into large particles upon chewing ${ }^{84}$ before melting in mouth. ${ }^{68}$ In addition to degradation of structure, melting of gelatine increases lubrication of foods. ${ }^{54}$ Often, fracture properties and melting properties of hydrocolloid gels are intertwined, but melting gels were shown to be perceived creamier and more melting than other gels with similar fracture properties. ${ }^{58}$ Even though friction properties of agar/gelatine gels decreased with melting, friction remained higher in those gels than in other gels that did not melt. Therefore, mechanical breakdown by melting is expected to contribute to a larger extent to the melting sensation than friction. In conclusion, melting is a dynamic phenomenon contributing to bolus formation that increases creaminess perception of fat containing foods towards the end of oral processing, as illustrated in Fig. 4.

3.1.3. Bolus mechanical properties. Rheological properties of boli change constantly over eating time. Different methods can be used to measure the rheological properties of boli. ${ }^{19}$ Such methods include modified Texture Profile Analysis (TPA) of cereal boli, ${ }^{14}$ oscillatory small deformation rheology of bread and cheese boli, ${ }^{34,35,83}$ force-displacement mechanical testing of model cheese boli, ${ }^{82}$ back extrusion ${ }^{33}$ and shear rheology of meat boli. ${ }^{85}$ A test similar to modified TPA was performed by Devezeaux de Lavergne et al. and was referred to as Two Cycle Penetration Test (TCPT) with output measures that were defined specifically to describe the mechanical properties of food boli. ${ }^{36}$

Few studies have related mechanical bolus properties to texture perception. Slipperiness and compliance of food boli are two sensory properties that correlate to bolus rheology during swallowing. ${ }^{86}$ Perceived hardness of cereal boli was found to decrease together with mechanical hardness of the boli. ${ }^{14}$ In bread, density and Young's modulus were found to impact bolus properties and dynamic texture perception. Using TDS sensory trajectories, it could be seen that a dense bread was perceived differently at the beginning and middle of mastication than other breads (Fig. 2), but similarly at the end of mastication. Both storage and loss modulus of boli decreased during oral processing but remained significantly higher in boli from the dense bread compared to boli from two other breads, suggesting that a target bolus viscoelasticity is not required to trigger swallowing. ${ }^{34}$ However, a target value of consistency index in bread boli could be required before swallow. ${ }^{83}$ Saint-Eve et al. found that bolus properties of cheeses at swallow related to cheese mechanical properties and that differences in bolus properties could explain differences in texture perception between cheeses. ${ }^{35}$ They highlighted the interest of measuring dynamic bolus formation for a better understanding of texture perception.

Devezeaux de Lavergne et al. measured dynamic changes in bolus mechanical properties using TСРТ. ${ }^{36,59}$ The parameters from TCPT identified as most relevant were first peak force, adhesiveness and flowability. These parameters might be related to the hardness, stickiness to the teeth and ability of the bolus to flow after a chew, respectively. Overall, first peak force decreased with increasing eating time. Flowability increased with eating time reflecting the decrease in structure of the bolus and the increase in lubrication. Flowability seemed to converge to a similar value for all gels, suggesting a target value of flowability might be required for swallowing gel boli. ${ }^{36}$ Flowability was positively correlated to creaminess suggesting that mechanical boli properties, that depended on reduction of structure and increase of lubrication, correlated best with complex texture attributes perceived at the end of oral processing. Adhesiveness increased with oral processing time as saliva was incorporated into the boli, especially at the end of oral processing. ${ }^{73}$ Adhesiveness of the boli at the beginning of oral processing correlated with stickiness perception. ${ }^{36}$

The main relationships between the properties of the boli of model gels and texture perception are summarised in Table 2 .

\subsection{Impact of chewing behaviour on dynamic texture perception}

Eating behaviour is known to impact texture perception ${ }^{87}$ and constantly change due to a sensory feedback system. ${ }^{88}$ Therefore, understanding dynamic texture perception requires knowledge about dynamic oral processing physiology and behaviour. Mastication and salivation patterns largely depend on food properties. ${ }^{89}$ Next to differences in food properties, it was observed that differences between individuals impact oral processes. ${ }^{90}$

3.2.1. Link between food properties and eating behaviour. It was observed that chewing cycles and duration varied greatly between foods. ${ }^{91-93}$ Harder foods led to higher muscle activity, longer duration of mastication and more chewing cycles. $^{60,94-96}$ Gels and sausages with a high fracture stress were masticated longer and with a higher muscle activity than 
those with a low fracture stress. Fracture strain showed no impact on muscle activity and eating duration of gels. ${ }^{29,55,58,59}$ These results demonstrate that when masticating harder foods consumers adapt their oral processing behaviour by masticating with higher muscle activity.

Other properties of foods, such as shape and size, lubrication, adhesiveness, deformability and heterogeneity were also found to influence oral processing behaviour. ${ }^{55,71,88,97,98}$ Melting of gelatine gels, which decreased rapidly the degree of structure, resulted in a shorter eating time, ${ }^{58}$ but this was not systematically observed. ${ }^{59}$ Gels having serum release were masticated shorter as they were expected to be more lubricated and easily swallowed after shorter oral processing times, whereas oil release did not impact chewing duration. ${ }^{29,59}$

Mastication parameters depend not only on food properties, but they allow to predict sensory perception of food. In gels, it was demonstrated that oral processing parameters could be directly related to perception. Sensory hardness was correlated to muscle activity at first bite. Chew down sensory attributes were related to jaw opening velocity and cycle duration of the first five chewing cycles. After-feel sensory attributes were related to jaw movement amplitudes of the later chewing cycles. $^{55}$ Moreover, eating parameters supposedly depend on the sensory feedback loop and are expected to change as the bolus is formed. For instance, muscle activity was higher at the beginning than at the end of oral processing. ${ }^{99}$ This demonstrates that more muscle effort is required when the food is at its initial state than when it is orally processed and broken down into a bolus. Saliva content in the bolus positively correlated with perception of the attribute sticky and negatively with attributes slippery and moist. ${ }^{36}$ The incorporation of saliva into the bolus is known to increase moisture content and lubrication of the bolus, which should reduce dryness perception. However, a positive correlation can be found between a dry perception and high saliva addition. Such a correlation comes from the fact that dry foods require a higher addition of saliva to be safely swallowed. Such relationships are more difficult to evidence in commercial foods, where many physical properties are convoluted, but have been previously reported. Different commercially available cheeses were masticated for different time periods and eating duration was the parameter that influenced bolus properties and dynamic sensory perception the most. ${ }^{35}$

3.2.2. Variability between consumers. The variability between subjects adds to the differences observed in bolus properties at different stages of oral processing. Variability can originate from specific subject characteristics, such as age and gender, among others. Elderly rated nuts differently in stickiness than younger subjects when performing TDS. ${ }^{100}$ However, no differences in the bolus properties between the two groups were found. Elderly with different chewing efficiencies produced different meat boli suggesting that age alone is not the only factor to consider. ${ }^{96}$ Overall, the mechanisms of differences in dynamic perception between elderly and younger subjects still remain unclear for some texture attributes.
Oral processing time can differ considerably between groups of subjects and can influence bolus properties and dynamic texture perception. Devezeaux de Lavergne et al. selected two groups of young healthy subjects that differed largely in eating duration of a piece of sausage, "short duration eaters" and of "long duration eaters". ${ }^{73}$ Both groups displayed similar chewing behaviour. Therefore, both groups broke down the sausage at a similar speed but swallowed boli that differed considerably in level of breakdown. Due to the differences in bolus properties at swallow, the groups perceived different dominant sensations at the end of oral processing demonstrating that the variability in sensory perception between groups of consumers can be explained, in the case of sausages, by differences in oral processing behaviour.

We conclude that eating duration impacts sensory perception. Eating duration is crucial in determining the sequence of attributes that are perceived as dominant over time (beginning, middle, end of oral processing time) and in determining which attributes are perceived as dominant. Oral processing behaviour is adaptive as the subjects perceive the food during eating. The main relationships between chewing behaviour and texture perception are summarised in Table 2.

\section{Industrial implications and future research}

The dynamic aspects of texture perception were reviewed, including the aspects of dynamic sensory methods and monitoring changes in bolus properties and eating behaviour during oral processing. Moreover, several food properties were reviewed to identify the drivers of temporal perceptions. Such knowledge enables to design food textures more effectively, which can lead to the development of more enjoyable foods and the development of healthier products. Integrating the knowledge discussed above, the model shown in Fig. 5 was proposed. ${ }^{101}$ This model summarizes the complex interactions between food properties and oral processing. Oral processing depends on oral physiology and behaviour, which is constantly modified during eating through a sensory regulation system. Oral physiology and behaviour control food breakdown and bolus properties, which are key to dynamic texture perception. As a result, texture perception depends both on food properties and individual variability between subjects. Different parameters influence different texture attributes perceived at different stages of oral processing. In the following paragraphs, possible applications of this model are discussed.

\subsection{Developing foods for target groups of consumers}

Differences in eating behaviour and physiology impact dynamic texture perception. Such differences between groups of individuals should be taken into account when designing foods for target groups of consumers as shown in Fig. 5. For instance, the elderly population is known to show a decrease in jaw muscle force. ${ }^{96,102}$ Therefore, soft solid and solid foods may require structural modifications to remain in the elderly's 


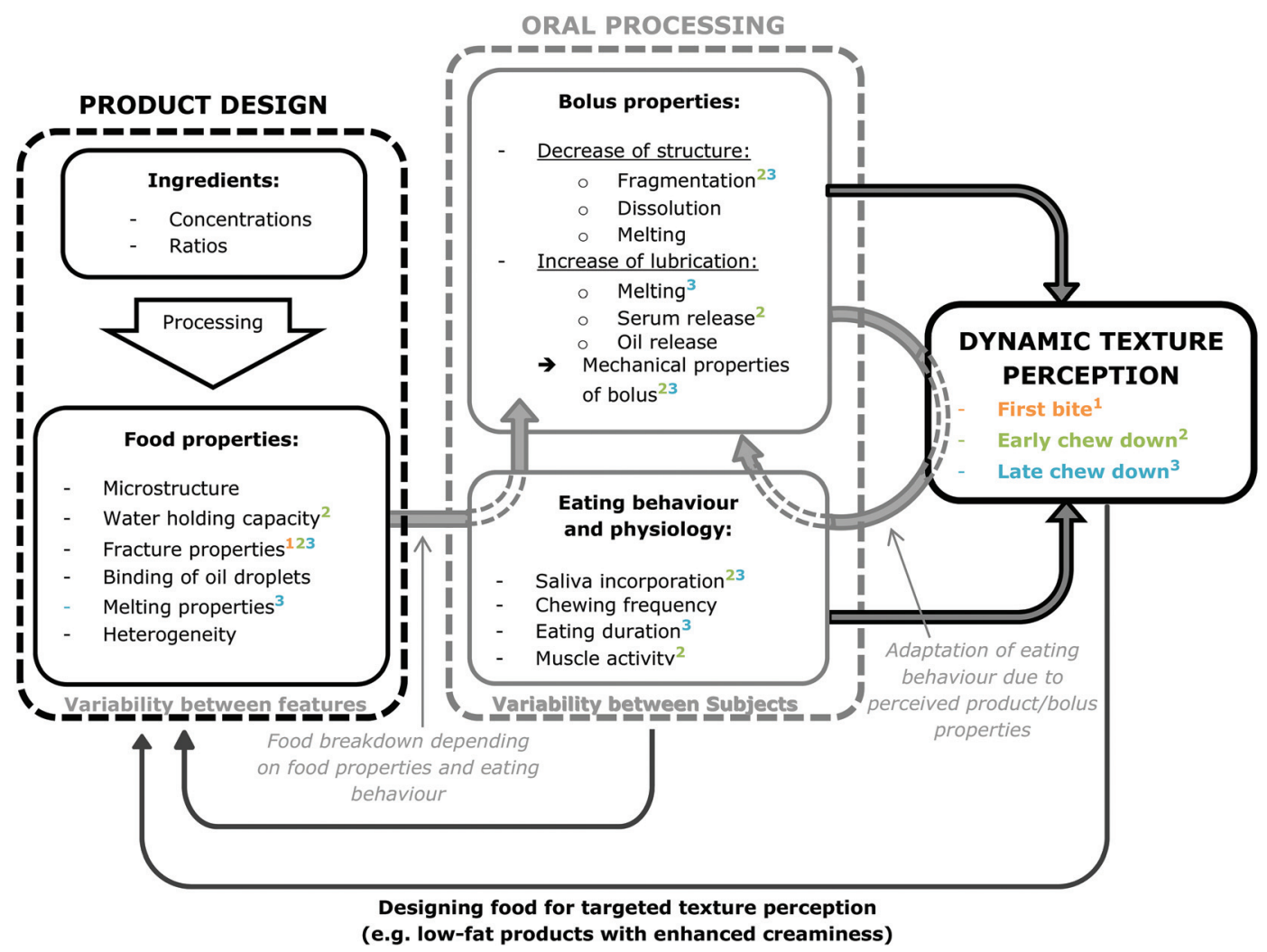

Fig. 5 Integration of product properties and oral processing in relation to dynamic texture perception and implications in food design. Superscript numbers indicate that parameters impact perception during (1) the early, (2) middle and (3) late chew down texture perception.

diet. ${ }^{103}$ Moreover, all elderly do not have the same physical and physiological condition and differences can be expected between segments of the elderly population. ${ }^{104}$ Next to that, the increasing demand for mass produced personalized foods represents an opportunity, yet a challenge, for developing new tailored textures. Four segments of consumers with different preferred foods textures have been proposed. ${ }^{105}$ Therefore, when targeting a specific group of consumers it is important to consider the eating behaviour of this group and their dynamic texture perception of the product. TDS would be a good sensory method in such research as it requires little training. ${ }^{44}$ Such an input when completed by the known preferences of the consumers could enable the design of a tailored texturized product for each consumer group.

\subsection{Developing foods contributing to a healthier diet: fat reduction}

Knowing the link between perception and food properties allows designing foods for an improved sensorial experience as shown in Fig. 5. One of the major texture attributes contributing to palatability of fat containing foods is creaminess. ${ }^{106}$ Moreover, fat content is being reduced in processed foods to answer to consumers demand for foods that might contribute to a healthier diet. However, palatability of food is often compromised by reformulations. ${ }^{12}$ Several product properties were linked to an improved creaminess and fattiness perception in emulsion-filled gels, for instance, fracture properties. Increasing fracture strain was shown to improve creaminess and fattiness independently from other gel properties. This was explained by the boli containing few, large, deformable particles as opposed to many small particles. Increasing fracture strain independently from fracture stress could enable to enhance fat related perception in foods allowing to reduce fat content while maintaining perception of fat related sensations. Also reducing fracture stress resulted in a higher creaminess. Such input shows the challenge of fat reduction. In cheeses, fat reduction was shown to increase fracture stress, ${ }^{49,107}$ but did not seem to change fracture strain. ${ }^{49}$ However, fracture mechanics of cheeses did not relate well to perception of chew down attributes. ${ }^{108}$ Increasing fracture strain of low fat cheeses might improve slightly creaminess, but the impact of increasing fracture strain compared to the impact of reducing fat content is yet unknown. In meat emulsions, fracture strain correlated with acceptability, ${ }^{109}$ which indicates fracture strain might be a highly relevant parameter to control while lowering fat content of such products.

Another major property that increases the perception of fat related attributes in model foods is melting of foods at mouth temperature. Melting increases the lubrication of the bolus and changes the mechanical properties of the bolus. Melting in mouth is known to create a desirable mouthfeel ${ }^{110}$ and to introduce dynamic changes in texture that increase 
palatability. ${ }^{111}$ Therefore, stabilizing an emulsion-filled gel with hydrocolloids that melt in mouth, such as gelatine, could be preferred. However, gelatine might be discarded as its consumption is restricted by vegetarian and religious lifestyle choices. Many gelling proteins or polysaccharides are used to replace gelatine, but none of them can replace fully the mouthfeel of gelatine. ${ }^{112}$

\section{Conclusions}

Texture perception is highly dynamic as it depends on constant manipulations and transformations of foods in the oral cavity. Therefore, the use of sensory methods that capture these dynamics is essential. In order to control texture perception several product properties can be varied and result in different breakdown pathways of foods. Mechanical properties govern the perception of texture attributes perceived at early stages of oral processing. Fracture properties are highly relevant in perception of first bite and chew down texture attributes. In addition, other food properties, such as melting and serum release, have a high impact on texture perception. All these food properties have to be monitored and can be controlled for designing desired food textures. Even though food properties are related to texture perception, knowing the properties of the bolus enables to unravel the link between food properties and dynamic texture perception. Fragmentation and changes in mechanical properties of the bolus contribute to the explanation of many texture attributes, including complex multimodal attributes such as creaminess. This knowledge is valuable input to understand the mechanisms linking food design to texture perception. In addition, eating behaviour is highly relevant for bolus formation and texture perception, and should be taken into account when designing food products for target consumer groups. With the trending topics of ageing population and personalized food products, effective food texture design is required.

\section{References}

1 A. S. Szczesniak, Texture is a sensory property, Food Qual. Prefer., 2002, 13, 215-225.

2 L. M. Barrangou, M. A. Drake, C. R. Daubert and E. A. Foegeding, Sensory texture related to large-strain rheological properties of agar/glycerol gels as a model food, J. Texture Stud., 2006, 37, 241-262.

3 K. C. Diehl and D. D. Hamann, Relationships between sensory profile parameters and fundamental mechanical parameters for raw potatoes, melons and apples, J. Texture Stud., 1980, 10, 401-420.

4 F. R. Harker, J. Maindonald, S. H. Murray, F. A. Gunson, I. C. Hallett and S. B. Walker, Sensory interpretation of instrumental measurements 1: Texture of apple fruit, Postharvest Biol. Technol., 2002, 224, 225-239.
5 L. van den Berg, T. van Vliet, E. van der Linden, M. A. J. S. van Boekel and F. van de Velde, Breakdown properties and sensory perception of whey proteins/polysaccharide mixed gels as a function of microstructure, Food Hydrocolloids, 2007, 21, 961-976.

6 G. Sala, G. A. van Aken, M. A. C. Stuart and F. van de Velde, Effect of droplet-matrix interactions on large deformation properties of emulsion-filled gels, J. Texture Stud., 2007, 38, 511-535.

7 E. Çakır, C. R. Daubert, M. Drake, C. J. Vinyard, G. Essick and A. E. Foegeding, The effect of microstructure on the sensory perception and textural characteristics of whey protein/k-carrageenan mixed gels, Food Hydrocolloids, 2011, 26, 33-43.

8 E. A. Foegeding, C. R. Daubert, M. A. Drake, G. Essick, M. Trulsson, C. Vinyard and F. van de Velde, A comprehensive approach to understanding textural properties of semi- and soft-solid foods, J. Texture Stud., 2011, 42, 103129.

9 K. Hiiemae, Mechanisms of food reduction, transport and deglutition: How the texture of food affects feeding behavior, J. Texture Stud., 2004, 35, 171-200.

10 J. B. Hutchings and P. J. Lillford, The perception of food texture - The philosophy of the breakdown path, J. Texture Stud., 1988, 19, 103-115.

$11 \mathrm{~J}$. Chen, Food oral processing-A review, Food Hydrocolloids, 2009, 23, 1-25.

12 C. Wilkinson, G. B. Dijksterhuis and M. Minekus, From food structure to texture, Trends Food Sci. Technol., 2000, 11, 442-450.

13 F. Mowlana and R. Heath, Assessment of masticatory efficiency: new methods appropriate for clinical research in dental practice, Eur. J. Prosthodontics Res. Dent., 1993, 1, 121-125.

14 M. A. Peyron, I. Gierczynski, C. Hartmann, C. Loret, D. Dardevet, N. Martin and A. Woda, Role of Physical Bolus Properties as Sensory Inputs in the Trigger of Swallowing, PLoS One, 2011, 6, e21167.

15 J. Chen, K. Niharika, L. Zhenyu and F. Takahiro, Influences of food hardness on the particle size distribution of food boluses, Arch. Oral Biol., 2013, 58, 293298.

16 C. Tournier, M. Grass, D. Zope, C. Salles and D. Bertrand, Characterization of bread breakdown during mastication by image texture analysis, J. Food Eng., 2012, 113, 615-622.

17 C. Loret, M. Walter, N. Pineau, M. A. Peyron, C. Hartmann and N. Martin, Physical and related sensory properties of a swallowable bolus, Physiol. Behav., 2011, 104, 855-864.

18 R. A. de Wijk, J. F. Prinz and A. M. Janssen, Explaining perceived oral texture of starch-based custard desserts from standard and novel instrumental tests, Food Hydrocolloids, 2006, 20, 24-34.

19 M. Panouillé, A. Saint-Eve and I. Souchon, Instrumental methods for bolus characterization during oral processing to understand food perceptions, Curr. Opin. Food Sci., 2016, 9, 42-49. 
20 E. A. Foegeding, C. J. Vinyard, G. Essick, S. Guest and C. Campbell, Transforming structural breakdown into sensory perception of texture, J. Texture Stud., 2015, 46, 152-170.

21 A. S. Szczesniak, Classification of textural characteristics, J. Food Sci., 1963, 28, 385-389.

22 M. A. Brandt, E. Z. Skinner and J. A. Coleman, Texture profile Method, J. Food Sci., 1963, 28, 404-409.

23 Y. Pascua, H. Koç and E. A. Foegeding, Food Structure: Roles of mechanical properties and oral processing in determining sensory texture of soft materials, Curr. Opin. Colloid Interface Sci., 2013, 18, 324-333.

24 H. Stone and J. L. Sidel, Descriptive analysis, in Sensory evaluation practices, ed. H. Stone and J. L. Sidel, Academic Press, London, 1985, p. 311.

25 M. Cliff and H. Heymann, Development and use of timeintensity methodology for sensory evaluation: A review, Food Res. Int., 1993, 26, 375-385.

26 G. B. Dijksterhuis and J. R. Piggott, Dynamic methods of sensory analysis, Trends Food Sci. Technol., 2000, 11, 284-290.

27 W. E. Lee and M. Pangborn, Time-intensity: the temporal aspects of sensory perception, Food Technol., 1986, 40, 71-78.

28 F. R. Jack, J. R. Piggoll and A. Paterson, Analysis of Textural Changes in Hard Cheese during Mastication by Progressive Profiling, J. Food Sci., 1994, 59, 539-543.

29 M. Devezeaux de Lavergne, M. van Delft, F. van de Velde, M. A. J. S. van Boekel and M. Stieger, Dynamic texture perception and oral processing of semi-solid food gels: Part 1: A comparison between QDA, progressive profiling and TDS, Food Hydrocolloids, 2015, 43, 207-217.

30 N. Pineau, P. Schlich, S. Cordelle, C. Mathonnière, S. Issanchou, A. Imbert, M. Rogeaux, P. Etiévant and E. Köster, Temporal Dominance of Sensations: Construction of the TDS curves and comparison with time-intensity, Food Qual. Prefer., 2009, 20, 450-455.

31 N. Pineau, A. G. De Bouillé, M. Lepage, F. Lenfant, P. Schlich, N. Martin and A. Rytz, Temporal Dominance of Sensations: What is a good attribute list?, Food Qual. Prefer., 2012, 26, 159-165.

32 F. Lenfant, C. Loret, N. Pineau, C. Hartmann and N. Martin, Perception of oral food breakdown. The concept of sensory trajectory, Appetite, 2009, 52, 659-667.

33 A. K. Young, J. N. Cheong, D. I. Hedderley, M. P. Morgenstern and B. J. James, Understanding the Link between Bolus Properties and Perceived Texture, J. Texture Stud., 2013, 44, 376-386.

34 M. Panouillé, A. Saint-Eve, I. Déléris, F. Le Bleis and I. Souchon, Oral processing and bolus properties drive the dynamics of salty and texture perceptions of bread, Food Res. Int., 2014, 62, 238-246.

35 A. Saint-Eve, M. Panouillé, C. Capitaine, I. Déléris and I. Souchon, Dynamic aspects of texture perception during cheese consumption and relationship with bolus properties, Food Hydrocolloids, 2015, 46, 144-152.
36 M. Devezeaux de Lavergne, F. van de Velde, M. A. J. S. van Boekel and M. Stieger, Dynamic texture perception and oral processing of semi-solid food gels: Part 2: Impact of breakdown behaviour on bolus properties and dynamic texture perception, Food Hydrocolloids, 2015, 49, 61-72.

37 J. C. Castura, L. Antúnez, A. Giménez and G. Ares, Temporal Check-All-That-Apply (TCATA): A novel dynamic method for characterizing products, Food Qual. Prefer., 2016, 47, 79-90.

38 G. Ares, J. C. Castura, L. Antúnez, L. Vidal, A. Giménez, B. Coste, A. Picallo, M. K. Beresford, S. L. Chheang and S. R. Jaeger, Comparison of two TCATA variants for dynamic sensory characterization of food products, Food Qual. Prefer., 2016, 54, 160-172.

39 A. K. Baker, J. C. Castura and C. F. Ross, Temporal CheckAll-That-Apply Characterization of Syrah Wine, J. Food Sci., 2016, 81(6), S1521-S1529.

40 G. Ares, S. R. Jaeger, L. Antúnez, L. Vidal, A. Giménez, B. Coste, A. Picallo and J. C. Castura, Comparison of TCATA and TDS for dynamic sensory characterization of food products, Food Res. Int., 2015, 78, 148-158.

41 D. Labbe, P. Schlich, N. Pineau, F. Gilbert and N. Martin, Temporal dominance of sensations and sensory profiling: A comparative study, Food Qual. Prefer., 2009, 20, 216-221.

42 M. Ng, J. B. Lawlor, S. Chandra, C. Chaya, L. Hewson and J. Hort, Using quantitative descriptive analysis and temporal dominance of sensations analysis as complementary methods for profiling commercial blackcurrant squashes, Food Qual. Prefer., 2012, 25, 121-134.

43 H. T. Lawless and H. Heymann, Sensory Evaluation of Food: Principles and Practices, Springer, New York, 2010.

44 J. N. Cheong, K. D. Foster, M. P. Morgenstern, J. M. V. Grigor, J. E. Bronlund, S. C. Hutchings and D. I. Hedderley, The application of temporal dominance of sensations (TDS) for oral processing studies: an initial investigation, J. Texture Stud., 2014, 45, 409-419.

45 S. Zorn, F. Alcaire, L. Vidal, A. Giménez and G. Ares, Application of multiple-sip temporal dominance of sensations to the evaluation of sweeteners, Food Qual. Prefer., 2014, 36, 135-143.

46 L. van den Berg, T. Van Vliet, E. Van der Linden, M. A. J. S. van Boekel and F. van de Velde, Physical properties giving the sensory perception of whey proteins/polysaccharide gels, Food Biophys., 2008, 3, 198-206.

47 L. van den Berg, A. L. Carolas, T. van Vliet, E. van der Linden, M. A. J. S. van Boekel and F. van de Velde, Energy storage controls crumbly perception in whey proteins/ polysaccharide mixed gels, Food Hydrocolloids, 2008, 22, 1404-1417.

48 R. Xiong, J. F. Meullenet, J. A. Hankins and W. K. Chung, Relationship between sensory and instrumental hardness of commercial cheeses, J. Food Sci., 2002, 67, 877-883.

49 E. A. Gwartney, E. A. Foegeding and D. K. Larick, The texture of commercial full-fat and reduced-fat cheese, J. Food Sci., 2002, 67, 812-816. 
$50 \mathrm{H}$. Wium, K. B. Qvist and M. Gross, Uniaxial compression of UF-feta cheese related to sensory texture analysis, J. Texture Stud., 1997, 28, 455-476.

51 E. H. J. Kim, V. K. Corrigan, A. J. Wilson, I. R. Waters, D. I. Hedderley and M. P. Morgenstern, Fundamental fracture properties associated with sensory hardness of brittle solid foods, J. Texture Stud., 2012, 43, 49-62.

52 L. M. Barrangou, M. A. Drake, C. R. Daubert and E. A. Foegeding, Textural properties of agarose gels. II. Relationships between rheological properties and sensory texture, Food Hydrocolloids, 2006, 20, 196-203.

53 E. A. Gwartney, D. K. Larick and E. A. Foegeding, Sensory texture and mechanical properties of stranded and particulate whey protein emulsion gels, J. Food Sci., 2004, 69, S333-S339.

54 G. Sala, R. A. de Wijk, F. van de Velde and G. A. van Aken, Matrix properties affect the sensory perception of emulsion-filled gels, Food Hydrocolloids, 2008, 22, 353-363.

55 E. Çakir, C. J. Vinyard, G. Essick, C. R. Daubert, M. Drake and A. E. Foegeding, Interrelations among physical characteristics, sensory perception and oral processing of protein-based soft-solid structures, Food Hydrocolloids, 2012, 29, 234-245.

56 H. Koç, C. J. Vinyard, G. K. Essick and E. A. Foegeding, Food Oral Processing: Conversion of Food Structure to Textural Perception, Annu. Rev. Food Sci. Technol., 2013, 4, 237-266.

57 G. Sala, Food gels filled with emulsion droplets: linking large deformation properties to sensory perception, $\mathrm{PhD}$ Thesis, 2007.

58 M. Devezeaux de Lavergne, V. M. G. Strijbosch, A. W. M. Van den Broek, F. Van de Velde and M. Stieger, Uncoupling the Impact of Fracture Properties and Composition on Sensory Perception of Emulsion-Filled Gels, J. Texture Stud., 2016, 47, 92-111.

59 M. Devezeaux de Lavergne, C. Tournier, D. Bertrand, C. Salles, F. van de Velde and M. Stieger, Dynamic texture perception, oral processing behaviour and bolus properties of emulsion-filled gels with and without contrasting mechanical properties, Food Hydrocolloids, 2016, 52, 648-660.

60 K. D. Foster, A. Woda and M. A. Peyon, Effect of texture of plastic and elastic model foods on the parameters of mastication, J. Neurophysiol., 2006, 95, 3469-3479.

61 S. Camacho, K. Liu, A. van der Linden, M. Stieger and F. J. van de Velde, J. Texture Stud., 2015, 46, 399-410.

62 L. van den Berg, T. van Vliet, E. van der Linden, M. A. J. S. van Boekel and F. van de Velde, Serum release: The hidden quality in fracturing composites, Food Hydrocolloids, 2007, 21, 420-432.

63 V. Urbonaite, H. H. J. de Jongh, E. van der Linden and L. Pouvreau, Origin of Water Loss from Soy Protein Gels, J. Agric. Food Chem., 2014, 62, 7550-7558.

64 G. Sala, F. van de Velde, M. A. C. Stuart and G. A. van Aken, Oil droplet release from emulsion-filled gels in relation to sensory perception, Food Hydrocolloids, 2007, 21, 977-985.

65 K. Liu, M. Stieger, E. van der Linden and F. van de Velde, Fat droplet characteristics affect rheological, tribological and sensory properties of food gels, Food Hydrocolloids, 2015, 44, 244-259.

66 E. Dickinson, Emulsion gels: The structuring of soft solids with protein-stabilized oil droplets, Food Hydrocolloids, 2012, 28, 224-241.

67 A. M. Janssen, M. E. J. Terpstra, R. A. de Wijk and J. F. Prinz, Relations between rheological properties, saliva-induced structure breakdown and sensory texture attributes of custards, J. Texture Stud., 2007, 38, 42-69.

68 Y. Inoue, M. Sasai, S. Shiga and H. Moritaka, Effects of Gel Amount and Mastication on the Velocity of Agar and Gelatin Gels Passing through the Pharynx, J. Jpn. Soc. Food Sci. Technol., 2009, 56, 261-270.

69 A. C. Mosca and J. Chen, Food oral management: physiology and objective assessment, Curr. Opin. Food Sci., 2016, 9, 11-20.

70 Q. Guo, A. Ye, M. Lad, D. Dalgleish and H. Singh, The breakdown properties of heat-set whey protein emulsion gels in the human mouth, Food Hydrocolloids, 2013, 33, 215-224.

71 S. C. Hutchings, K. D. Foster, J. E. Bronlund, R. G. Lentle, J. R. Jones and M. P. Morgenstern, Mastication of heterogeneous foods- Peanuts inside two different food matrices, Food Qual. Prefer., 2011, 22, 332-339.

72 A. C. Mosca, F. Van de Velde, J. H. F. Bult, M. A. J. S. Van Boekel and M. Stieger, Effect of gel texture and sucrose spatial distribution on sweetness perception, LWT-Food Sci. Technol., 2012, 46, 183-188.

73 M. Devezeaux de Lavergne, J. A. M. Derks, E. C. Ketel, R. A. de Wijk and M. Stieger, Eating behaviour explains differences between individuals in dynamic texture perception of sausages, Food Qual. Prefer., 2015, 41, 189-200.

74 D. S. Larsen, J. Tang, L. Ferguson, P. M. Morgenstern and B. J. James, Oral breakdown of texturally complex gelbased model food, J. Texture Stud., 2015, 47, 169-180.

75 S. A. Rodrigues, A. K. Young, B. J. James and M. P. Morgenstern, Structural changes within a biscuit bolus during mastication, J. Texture Stud., 2014, 45, 89-96.

76 P. Lillford, The importance of food microstructure in fracture physics and texture perception, J. Texture Stud., 2011, 42, 130-136.

77 G. Sala and M. Stieger, Time to first fracture affects sweetness of gels, Food Hydrocolloids, 2013, 30, 73-81.

78 J. S. Chen and L. Lolivret, The determining role of bolus rheology in triggering a swallowing, Food Hydrocolloids, 2011, 25, 325-332.

79 A. Tarrega, C. Yven, E. Semon and C. Salles, In-mouth aroma compound release during cheese consumption: Relationship with food bolus formation, Int. Dairy J., 2011, 21, 358-364. 
80 J. H. H. Bongaerts, D. Rossetti and J. R. Stokes, The Lubricating Properties of Human Whole Saliva, Tribol. Lett., 2007, 27, 277-287.

81 C. Pradal and J. R. Stokes, Oral tribology: bridging the gap between physical measurements and sensory experience, Curr. Opin. Food Sci., 2016, 9, 34-41.

82 S. R. Drago, M. Panouillé, A. Saint-Eve, E. Neyraud, G. Feron and I. Souchon, Relationships between saliva and food bolus properties from model dairy products, Food Hydrocolloids, 2011, 25, 659-667.

83 F. Le Bleis, L. Chaunier, G. Della Valle, M. Panouille and A. L. Reguerre, Physical assessment of bread destructuration during chewing, Food Res. Int., 2013, 50, 308-317.

84 A. M. Muñoz, R. M. Pangborn and A. C. Noble, Sensory and mechanical attributes of gels texture. 2. Gelatin, sodium alginate and k-carrageenan gels, J. Texture Stud., 1986, 17, 17-36.

85 C. Yven, J. Culioli and L. Mioche, Meat bolus properties in relation with meat texture and chewing context, Meat Sci., 2005, 70, 365-371.

86 H. S. Seo, I. K. Hwang, T. R. Han and I. S. Kim, Sensory and instrumental analysis for slipperiness and compliance of food during swallowing, J. Food Sci., 2007, 72, 707-713.

87 W. E. Brown, K. R. Langley, A. Martin and H. J. H. MacFie, Characterisation of patterns of chewing behaviour in human subjects and their influence on texture perception, J. Texture Stud., 1994, 25, 455-468.

88 A. van der Bilt, H. W. van der Glas, L. W. Olthoff and F. Bosman, The effect of particle size reduction on the jaw gape in human mastication, J. Dent. Res., 1991, 70, 931937.

89 K. K. Kapur, N. R. Garrett and E. Fischer, Effects of anaesthesia of human oral structures on masticatory performance and food particle size distribution, Arch. Oral Biol., 1990, 35, 397-403.

90 A. Woda, K. Foster, A. Mishellany and M. Peyron, Adaptation of healthy mastication to factors pertaining to the individual or to the food, Physiol. Behav., 2006, 89, 2835.

91 K. Hiiemae, M. R. Heath, G. Heath, E. Kazazoglu, J. Murray, D. Sapper and K. Hamblett, Natural bites, food consistency and feeding behaviour in man, Arch. Oral Biol., 1996, 41, 175-189.

92 C. E. Wilson and W. E. Brown, Influence of food matrix structure and oral breakdown during mastication on temporal perception of flavour, J. Sens. Stud., 1997, 12, 69-86.

93 F. A. Fontijn-Tekamp, A. van der Bilt, J. H. Abbink and F. Bosman, Swallowing threshold and masticatory performance in dentate adults, Physiol. Behav., 2004, 83, 431436.

94 M. A. Peyron, C. Lassauzay and A. Woda, Effects of increased hardness on jaw movement and muscle activity during chewing of visco-elastic model foods, Exp. Brain Res., 2002, 142, 41-51.
95 M. A. Peyron, O. Blanc, J. P. Lund and A. Woda, Influence of age on adaptability of human mastication, J. Neurophysiol., 2004, 92, 773-779.

96 L. Mioche, Mastication and food texture perception: Variation with age, J. Texture Stud., 2004, 35, 145-158.

97 L. Engelen, A. van der Bilt, M. Schipper and F. Bosman, Oral size perception of particles: Effect of size, type, viscosity and method, J. Texture Stud., 2005, 36, 373-386.

98 H. Koç, E. Çakir, C. J. Vinyard, G. Essick, C. R. Daubert, M. A. Drake, J. Osborne and E. A. Foegeding, Adaptation of oral processing to the fracture properties of soft solids, J. Texture Stud., 2013, 45, 47-61.

99 H. Iguchi, J. Magara, Y. Nakamura, T. Tsujimura, K. Ito and $\mathbf{M}$. Inoue, Changes in jaw muscle activity and the physical properties of foods with different textures during chewing behaviors, Physiol. Behav., 2015, 152, 217-224.

100 S. C. Hutchings, K. D. Foster, D. I. Hedderley and M. P. Morgenstern, Differences between Age Groups in the Use of the Temporal Dominance of Sensations Technique across a Range of Food Textures, J. Texture Stud., 2014, 45, 206-219.

101 M. Devezeaux de Lavergne, Bolus matters: impact of food oral breakdown on dynamic texture perception, $\mathrm{PhD}$ Thesis, 2015.

102 K. Kohyama, L. Mioche and J. F. Martin, Chewing patterns of various texture foods studied by electromyography in young and elderly populations, J. Texture Stud., 2002, 33, 269-283.

103 M. Peleg, Tailoring texture for the elderly: Theoretical aspects and technological options, Crit. Rev. Food Sci. Nutr., 1993, 33, 45-55.

104 A. Mishellany-Dutour, J. Renaud, M. A. Peyron, F. Rimek and A. Woda, Is the goal of mastication reached in young dentates, aged dentates and aged denture wearers?, Br. J. Nutr., 2008, 99, 121-128.

105 M. A. Jeltema, J. B. Beckley and J. Vahalik, Importance of Understanding Mouth Behavior when Optimizing Product Texture now and in the future, in Food texture design and optimization, ed. Y. Dar and J. Light, Wiley \& Sons, Ltd., West Sussex, U.K., 2014, pp. 423-442.

106 R. A. de Wijk, M. E. J. Terpstra, A. M. Janssen and J. F. Prinz, Perceived creaminess of semi-solid foods, Trends Food Sci. Technol., 2006b, 17, 412-422.

107 T. P. Guinee, M. A. E. Auty and M. A. Fenelon, The effect of fat content on the rheology, microstructure and heatinduced functional characteristics of Cheddar cheese, Int. Dairy J., 2000, 10, 277-288.

108 E. A. Foegeding, J. Brown, M. Drake and C. R. Daubert, Sensory and mechanical aspects of cheese texture, Int. Dairy J., 2003, 13, 585-591.

109 E. Tornberg, K. Andersson, A. Andersson and A. Josell, The texture of comminuted meat products, Food Aust., 2000, 52, 519-524.

110 F. Hayakawa, Y. Kazami, S. Ishihara, S. Nakao, M. Nakauma, T. Funami, K. Nishinari and K. Kohyama, 
Characterization of eating difficulty by sensory evaluation of hydrocolloid gels, Food Hydrocolloids, 2014, 38, 95-103.

111 R. J. Hyde and S. A. Witherly, Dynamic contrast: a sensory contribution to palatability, Appetite, 1993, 21, 1-16.
112 I. J. Haug, K. I. Draget and O. Smidsrød, Physical and rheological properties of fish gelatin compared to mammalian gelatin, Food Hydrocolloids, 2004, 18, 203-213. 